Preprint JINR E2-95-383

hep-th/9508137

\title{
Quantum oscillator and a bound system of two dyons
}

\author{
V. M. Ter-Antonyan [, A. Nersessian i \\ Bogoliubov Laboratory of Theoretical Physics, \\ Joint Institute for Nuclear Research, \\ Dubna, Moscow Region, 141980, Russia
}

\begin{abstract}
It is shown that $U(1)$-Hamiltonian reduction of a four-dimensional isotropic quantum oscillator results in a bound system of two spinless Schwinger's dyons. Its wavefunctions and spectrum are constructed.
\end{abstract}

*e-mail:terant@thsun1.jinr.dubna.su

†e-mail:nerses@thsun1.jinr.dubna.su 


\section{Introduction}

In our paper [4] a classical integrable bound system describing the non-relativistic interaction of a spinless particle with the $U(1)$-dyon in the centre-of-mass system ("chargedyon" system) was constructed by the $U(1)$ Hamiltonian reduction of an oscillator on the twistor space. This system is specified by hidden symmetry caused by the Runge-Lenz vector- like constants of motion. It describes not only the "charge-dyon" interaction, but also the "dyon-dyon" one.

In the present paper, by an analogous reduction at the quantum level, we construct the Schrödinger equation, constants of motion and wave functions and spectrum for a non-relativistic bound system describing the interaction of two Schwinger dyons in the center-of-mass system. Despite simplicity, this system inherits such remarkable properties of monopole systems (see, e.g. [3] and refs therein), as a degenerate ground state and "spin transmutation". A quantum system, unlike from a classical one, explicitly depends from the vector potential of a monopole field. It was shown in the Zwanziger paper [1] that only the system, specified by the vector potential of the Schwinger monopole, can be interpreted as the "dyon-dyon" one, while similar system with the vector potential of the Dirac monopole characterizes only the "charge-dyon" interaction. Notice that such a quantum system, depending on the Dirac monopole vector potential has been constructed by a similar reduction in ref. [5]. However, the authors did not know the physical meaning of the system they constructed, therefore, its important properties were unnoticed [6]. In our consideration, we will omit the details of calculation coincident with those of classical reduction [4].

We will use the following notation: $\mu$ and $\omega$ are oscillator parameters; $z^{\alpha}=u^{\alpha}+i u^{\alpha+2}$ and $\vec{r}=\left(x^{1}, x^{2}, x^{3}\right)$ are Cartesian coordinates of spaces $\mathbb{C}^{2}=\mathbb{R}^{4}$ and $\mathbb{R}^{3}$ respectively, $u=|z|, r=|\vec{r}| ; \vec{\sigma}$ are the Pauli matrices in a standard representation; and $a=\left(\frac{\mu \omega}{\hbar}\right)^{\frac{1}{2}}$ is a parameter with the dimension of inverse length.

\section{The Schrïodinger equation and constants of motion}

An isotropic oscillator on the space $\mathbb{C}^{2}$ is described by the Schrörodinger's equation

$$
\frac{\partial^{2} \Psi}{\partial z^{\alpha} \partial \bar{z}^{\alpha}}+\frac{2 \mu}{\hbar^{2}}\left[\frac{E}{4}-\frac{\mu \omega^{2} z \bar{z}}{8}\right] \Psi=0 \quad \Leftrightarrow \quad \mathcal{H}_{o s c} \Psi=E \Psi .
$$

Its constants of motion

$$
\mathcal{J}_{i}=\frac{1}{2}\left(z^{\alpha} \sigma_{\beta \alpha}^{i} \frac{\partial}{\partial z^{\beta}}-\bar{z}^{\alpha} \sigma_{\alpha \beta}^{i} \frac{\partial}{\partial \bar{z}^{\beta}}\right), \quad \mathcal{I}_{i}=-4 \frac{\hbar^{2}}{2 \mu} \sigma_{\alpha \beta}^{i} \frac{\partial}{\partial z^{\beta}} \frac{\partial}{\partial \bar{z}^{\alpha}}+\frac{\mu \omega^{2}}{2} \sigma_{\alpha \beta}^{i} z^{\alpha} \bar{z}^{\beta},
$$

form the algebra:

$$
\left\{\mathcal{J}_{k}, \mathcal{J}_{l}\right\}=i \varepsilon_{k l m} \mathcal{J}_{m}, \quad\left\{\mathcal{J}_{k}, \mathcal{I}_{l}\right\}=i \varepsilon_{k l m} \mathcal{I}_{m}, \quad\left\{\mathcal{I}_{k}, \mathcal{I}_{l}\right\}=i(2 \hbar \omega)^{2} \varepsilon^{k l m} \mathcal{J}_{m}
$$

Let us now perform the quantum reduction of this system with respect to the action of group $U(1)$ given by the operator

$$
\mathcal{J}_{0}=\frac{1}{2}\left(z^{\alpha} \frac{\partial}{\partial z^{\alpha}}-\bar{z}^{\alpha} \frac{\partial}{\partial \bar{z}^{\alpha}}\right),
$$


commuting with the constants of motion (2) and with the oscillator's Hamiltonian

$$
\left[\mathcal{J}_{0}, H_{\text {osc }}\right]=0, \quad\left[\mathcal{J}_{0}, \mathcal{J}_{i}\right]=0 \quad\left[\mathcal{J}_{0}, \mathcal{I}_{i}\right]=0
$$

To this end, we introduce the operators

$$
\vec{r}=\bar{z}^{\alpha} \vec{\sigma}_{\alpha \beta} z^{\beta}, \quad \hat{\vec{p}}=\frac{i \hbar}{2(z \bar{z})}\left(z^{\alpha} \hat{\sigma}_{\beta \alpha} \frac{\partial}{\partial z^{\beta}}+\bar{z}^{\alpha} \hat{\sigma}_{\alpha \beta} \frac{\partial}{\partial \bar{z}^{\beta}}\right)
$$

obeying the relations

$$
\begin{aligned}
{\left[\mathcal{J}_{0}, \vec{r}\right] } & =0, \quad\left[\mathcal{J}_{0}, \hat{\vec{p}}\right]=0 \\
{\left[x^{k}, x^{l}\right]=0, \quad\left[x^{k}, \hat{p}^{l}\right] } & =i \hbar \delta^{k l}, \quad\left[\hat{p}^{k}, \hat{p}^{l}\right]=\hbar^{2} \varepsilon^{k l m} \frac{x^{m}}{r^{3}} \mathcal{J}_{0} .
\end{aligned}
$$

To a fixed eigenvalue of the operator $\mathcal{J}_{0}$

$$
\mathcal{J}_{0} \Psi=s \Psi
$$

there corresponds the wavefunction

$$
\Psi(z, \bar{z})=\psi(\vec{r}) e^{i s \gamma}
$$

where $\gamma$ is a function conjugate to the operator $\mathcal{J}_{0}$ :

$$
\gamma=\frac{i}{2}\left(\log z^{1} / \bar{z}^{1}+\log z^{2} / \bar{z}^{2}\right), \quad \gamma \in[0,4 \pi): \quad\left[\mathcal{J}_{0}, \gamma\right]=i
$$

Here also we have

$$
\hat{\vec{p}}\left(\psi e^{i s \gamma}\right)=(\hat{\pi} \psi) e^{i s \gamma}
$$

where

$$
\hat{\pi}=-i \hbar \frac{\partial}{\partial \vec{r}}-\hbar s \vec{A}, \quad \vec{A}=\frac{\vec{n}_{3} \vec{r}}{r} \frac{\vec{n}_{3} \times \vec{r}}{r^{2}-\left(\vec{n}_{3} \vec{r}\right)^{2}}
$$

and $\vec{A}$ is a vector potential of the Schwinger's monopole with a unit magnetic charge and a singular line along the axis $x^{3} ; \vec{n}_{3}=(0,0,1)$.

Owing to the relations (5) and (7), the oscillator's Hamiltonian and constants of motion (2) are expressed through $\vec{r}, \hat{\vec{p}}, \mathcal{J}_{0}$. As a result, the substitution (10) reduces equation (四) to the form

$$
\hat{H} \psi=-\frac{\mu \omega^{2}}{8} \psi, \quad \hat{H}=\frac{\hbar^{2}}{2 \mu} \hat{\pi}^{2}-\frac{E}{4 r}+\frac{\hbar^{2} s^{2}}{2 \mu r^{2}},
$$

whereas the constants of motion of the oscillator (2) are reduced to the operators

$$
\vec{J}=-\hat{\pi} \times \vec{r}+\frac{\hbar s \vec{r}}{r}, \quad \vec{I}=\frac{\hbar^{2}}{2 \mu} \hat{\pi} \times \vec{J}+\frac{\vec{r}}{2 r},
$$

i.e. to the total angular momentum of the system and to an analog of the Runge-Lenz vector.

From the requirement for the wavefunction (10) being single--valued we derive immediately

$$
s=0, \pm 1 / 2, \pm 1, \ldots
$$


The obtained system describes nonrelativistic interaction of two spinless dyons with electric amd magnetic charges $\left(e_{1}, g_{1}\right)$ and $\left(e_{2}, g_{2}\right)$ and energy $\mathcal{E}$ if we put

$$
\frac{e_{1} g_{2}-e_{2} g_{1}}{\hbar c}=s, \quad e_{1} e_{2}+g_{1} g_{2}=\frac{E}{4}, \quad \mathcal{E}=-\frac{\mu \omega^{2}}{8} .
$$

The parameter $\mu$ represents the reduced mass; the description holds in the centre-of-mass system [1]. The first of formulae (17) together with (16) acquires the meaning of the Dirac's condition of charge quantization.

Remark. The vector-potential shape in (13) depends on the choice of the coordinate $\gamma$ conjugate to the operator $\mathcal{J}_{0}$. For instance, the vector potential of the Dirac's monopole is described by the following choice of the coordinate $\gamma$ [5] :

$$
\gamma=i \log z^{1} / \bar{z}^{1}, \gamma \in[0,4 \pi) \Rightarrow \vec{A}=\frac{1}{r} \frac{\vec{n}_{3} \times \vec{r}}{r-\left(\vec{n}_{3} \vec{r}\right)} .
$$

This system can be interpreted only as the "charge-Dirac's dyon" system [4, 1].

\section{Wavefunctions and spectrum}

The system of equations

$$
\begin{array}{cl}
\mathcal{H}_{\text {osc }} \Psi=E \Psi & , \quad \mathcal{J}_{i} \mathcal{J}_{i} \Psi=j(j+1) \Psi \\
\mathcal{J}_{0} \Psi=s \Psi & , \quad \mathcal{J}_{3} \Psi=m \Psi,
\end{array}
$$

is separated in the coordinates $u \in[0, \infty), \beta \in[0, \pi], \alpha \in[0,2 \pi), \gamma \in[0,4 \pi)$ :

$$
z_{1}=u \cos \frac{\beta}{2} e^{-i \frac{\alpha+\gamma}{2}} \quad z_{2}=u \sin \frac{\beta}{2} e^{i \frac{\alpha-\gamma}{2}} .
$$

As a result, the solution to the system (19) is of the form

$$
\Psi_{E j m s}=R_{E j}(u) D_{m s}^{j}(\alpha, \beta, \gamma) .
$$

where $D_{m s}^{j}(\alpha, \beta, \gamma)$ is the Wigner function

$$
D_{m s}^{j}(\alpha, \beta, \gamma)=e^{i m \alpha} d_{m s}^{j}(\beta) e^{i s \gamma}
$$

and the radial function $R_{E j}$ obeys the equation

$$
\frac{d^{2} R_{E j}}{d \rho^{2}}+\frac{3}{\rho} \frac{d R_{E j}}{d \rho}-\left[\frac{4 j(j+1)}{\rho^{2}}+\rho^{2}-\lambda\right] R_{E j}=0
$$

with $\rho=(a u)^{2}, \lambda=\frac{2 \mu E}{\hbar^{2} a^{2}}$,

The substitution $R_{E j}=\rho^{j} \mathrm{e}^{-\rho / 2} W(\rho)$ reduces eq. (23) to the equation for the confluent hypergeometric function

$$
\rho W^{\prime \prime}+(2 j+1-\rho) W^{\prime}+\left(\frac{\lambda}{4}-j-1\right) W=0 .
$$


The solution regular at the point $\rho=0$ is given by

$$
W(\rho)=\operatorname{const} F\left(j+1-\frac{\lambda}{4}, 2 j+1, \rho\right) .
$$

As a result,

$$
R_{n j}(\rho)=\text { const } \rho^{j} \mathrm{e}^{-\rho / 2} F(-n+1,2 j+1, \rho),
$$

where $j+1-\lambda / 4=-n+1$. Expressions (21), (22) and (24) determine the oscillator basis.

From the requirement $R_{E j}(\infty)=0$ and uniqueness of the function (22)it follows, that: $n=1,2,3, \ldots ; m, s=-j,-j+1, \ldots, j-1, j ; 2 j=0,1, \ldots$ Then, upon introducing the principal quantum number $N=2 n+2 j-2$, we obtain the following relations for the oscillator spectrum:

$$
\begin{aligned}
& E=\hbar \omega(N+2), \quad N=0,1,2, \ldots ; \\
& 2 j=0,1, \ldots N \\
& m, s=-j,-j+1, \ldots, j-1, j .
\end{aligned}
$$

At fixed $j$ the $(2 j+1)^{2}$ states corresponds to the level $E_{N}$. Since $j=N / 2, N / 2-1, \ldots$, the degree of degeneracy of the $N$ th level is equals to

$$
g_{N}=\frac{1}{6}(N+1)(N+2)(N+3) \text {. }
$$

Now we can construct the wavefunctions and spectrum of the reduced system.

The coordinates of space $\mathbb{C}^{2}$ transform into the spherical coordinates of space $\mathbb{R}^{3}:(r=$ $\left.u^{2}, \theta=\beta, \phi=\alpha\right)$.

Comparison of (10) with (21) gives the following wavefunction of the reduced system

$$
\psi_{n j m}(\vec{r} ; s)=\text { const } R_{n j}(a r) d_{m s}^{j}(\theta) e^{i m \phi} .
$$

and expressions (17), (25) result in the energy spectrum for the system

$$
\mathcal{E}_{k}^{s}=-\frac{\mu\left(e_{1} e_{2}+g_{1} g_{2}\right)^{2}}{2 \hbar^{2}(k+|s|)^{2}}, \quad k=1,2, \ldots
$$

For fixed $\mathcal{E}_{k}^{s}$

$$
j=|s|,|s|+1, \ldots, k+|s|-1 ; \quad m=-j,-j+1, \ldots, j-1, j .
$$

Therefore, the energy levels (29) are degenerated with multiplicity $g_{k}^{s}=k(k+2|s|)$.

Thus, having reduced a 4-dimensional quantum oscillator, we have constructed the Schrörodinger's equation for a bound system of two Schwinger's dyons, its constants of motion, wavefunctions and the spectrum.

We stress that the quantum numbers $j, m$ characterize the total angular momentum (spin) and its projection onto the axis $x_{3}$. Therefore, integer and half-integer values of $s$ represent, respectively, integer and half- integer values of the system's spin . At $s=0$ the system becomes hydrogen-like. 
Under the identical transformation $\phi \rightarrow \phi+2 \pi$, the wavefunction of the reduced system acquires the phase $2 \pi m$ : it is single-valued at integer $s$ and changes in sign at half-integer $s$.

The wavefunction of the ground state $(k=1, j=|s|)$ of the system is of the form

$$
\psi_{1, m}(\vec{r} ; s)=\text { const } r^{|s|} e^{-r /(|s|+1)}(\sin \theta)^{|s|}\left(\tan \frac{\theta}{2}\right)^{\mp m} e^{i m \phi} .
$$

It is seen that the ground state is degenerated (with respect to the quantum number $m$ ) and is not spherically symmetric: the system has a nonzero dipole moment.

Note is to be made that when $m \neq \pm|s|$, we have $|\psi(\theta=0)|^{2}=|\psi(\theta=\pi)|^{2}=0$, which means that the system is flattened to the plane $x^{3}=0$ and the charge cannot be on the singular line. This property holds valid for excited states as well.

At $m=|s|$ we have $|\psi(\theta=0)|^{2} \neq 0,|\psi(\theta=\pi)|^{2}=0$, which implies that the charge cannot be on the lower semiaxis $x^{3}$. At $m=-|s|$ the charge cannot be on the upper semiaxis $x^{3}$.

Acknowledgments. The authors are thankful to L.Mardoyan for useful discussions and to V.I. Ogievetsky, A.I. Pashnev, A.N. Sissakian and Y.Uwano for interest in the work. The work of A.N. has been made possible by a fellowship of the Grant No. M21300 from International Science Foundation and INTAS Grant No. 93-2492 and have been carry out within the research program of the International Center of Fundamental Physics in Moscow.

\section{References}

[1] D. Zwanziger, Phys. Rev. 176 (1968), 1480 .

[2] J. Schwinger, Science 165 (1969), 757

[3] R. Jackiw, Ann. Phys. 129 (1980), 183

[4] A. Nersessian, V. Ter-Antonyan , Mod. Phys. Lett. A9 (1994), 2431

[5] T. Iwayi, Y. Uwano, J.Phys. A: Math. Gen., 21 (1988), 4083

[6] T. Iwayi, Y. Uwano, Private communication 